\title{
1 A parasite's paradise: Biotrophic species prevail oomycete 2 community composition in tree canopies
}

3 Running title: Biotrophic oomycetes in tree canopies

4 Robin-Tobias Jauss ${ }^{1^{*}}$, Susanne Walden ${ }^{2}$, Anna Maria Fiore-Donno ${ }^{2}$, Stefan Schaffer ${ }^{3,4}$,

5 Ronny Wolf ${ }^{3}$, Kai Feng ${ }^{2,5,6}$, Michael Bonkowski ${ }^{2}$, Martin Schlegel ${ }^{1,4}$

$6{ }^{1}$ University of Leipzig, Institute of Biology, Biodiversity \& Evolution, Talstraße 33, 04103 Leipzig

$72{ }^{2}$ University of Cologne, Institute of Zoology, Terrestrial Ecology, Zülpicher Straße 47b, 50674 Köln

$8{ }^{3}$ University of Leipzig, Institute of Biology, Molecular Evolution \& Animal Systematics, Talstraße 33, 04103 Leipzig

$9{ }^{4}$ German Centre for Integrative Biodiversity Research (iDiv) Halle Jena Leipzig, Deutscher Platz 5e, 04103 Leipzig

$10{ }^{5}$ CAS Key Laboratory for Environmental Biotechnology, Research Center for Eco-Environmental Sciences, Chinese Academy

11 of Sciences, Beijing, China

$12{ }^{6}$ College of Resources and Environment, University of Chinese Academy of Sciences, Beijing, China

13 *To whom correspondence should be addressed. E-mail: jauss@uni-leipzig.de

\section{Abstract}

15 Oomycetes (Stramenopiles, Protista) are among the most severe plant pathogens, 16 comprising species with a high economic and ecologic impact on forest ecosystems. Their 17 diversity and community structures are well studied in terrestrial habitats, but tree canopies 18 as huge and diverse habitats have been widely neglected. A recent study highlighted distinct 19 oomycete communities in the canopy region compared to forest soils when taking oomycete 20 abundances into account, in contrast to the homogeneity at the incidence level. It remains 21 however unknown if this homogeneity also leads to a functional homogenisation among 22 microhabitats. In this study, we supplemented functional traits to oomycete canopy and 23 ground communities, which were determined over a time period of two years with a 24 metabarcoding approach. Our results showed that even though most oomycetes occurred in 25 all habitats, a strong discrepancy between the strata and correspondingly the distribution of 
oomycete lifestyles could be observed, which was constant over time. Obligate biotrophic species, exclusively feeding on living host tissue, dominated the canopy region, implying tree canopies to be a hitherto neglected reservoir for parasitic protists. Parasites highly specialised on hosts that were not sampled could be determined in high abundances in the canopy and the surrounding air, challenging the strict host dependencies ruled for some oomycetes. Our findings further contribute to the understanding of oomycete ecosystem functioning in forest ecosystems.

Keywords: protists, oomycetes, canopies, metabarcoding, parasites, forest ecosystems

\section{INTRODUCTION}

35 Some of the most devastating plant pathogens with worldwide economic and ecologic 36 relevance belong to the Oomycota, protists in the Stramenopiles within the SAR 37 superkingdom (Adl et al., 2019). They comprise several distinct lineages, i.a. the Pythiales,

38 Peronosporales and Saprolegniales (Marano et al., 2014) and occupy ecologically important positions as saprotrophs and severe pathogens. The infamous oomycete Phytophthora

40 infestans causes one of the most destructive plant diseases, the potato late blight, and 41 initiated the great Irish famine in the late 1840's with a million deaths and massive 42 emigration (Mizubuti \& Fry, 2006). The ecological and economic impact of oomycetes has 43 led to an increased research interest on their community structures (Robideau et al., 2011;

44 Riit et al., 2016; Singer et al., 2016; Jauss et al., 2020b, 2020a; Fiore-Donno \& Bonkowski, 45 2021), and, correspondingly, their pathogenicity and infection strategies (Rizzo \& Garbelotto, 46 2003; Rizzo et al., 2005; Thines \& Kamoun, 2010).

47 Three lifestyles are described for oomycetes: Saprotrophic species are free-living and feed 48 on dead and decaying matter (Lewis, 1973). They occupy key roles in the trophic upgrading 49 of terrestrial, marine and freshwater habitats (Marano et al., 2016). Although saprotrophy is less common in oomycetes, it is believed to be the ancestral state of oomycete nutrition ( $F$. 
51 Martin et al., 2016; Spanu \& Panstruga, 2017), while the majority of currently described

52 oomycetes are plant pathogens (Thines \& Kamoun, 2010). The pathogenic lifestyles include

53 hemibiotrophy, characterised by an initial biotrophic phase later turning into a necrotrophic

54 phase after the death of the host (Fawke et al., 2015; Pandaranayaka et al., 2019), as well

55 as obligate biotrophy, which comprises species exclusively feeding on living host tissue

56 (Spanu \& Kämper, 2010). Even though obligate biotrophic species usually do not actively kill

57 their host, they still damage the host by chlorosis, inflorescence and killing of seedlings, and

58 thus cause severe economic losses (Parkunan et al., 2013; Krsteska et al., 2014; Kamoun et

59 al., 2015).

60 Oomycete communities are well studied in terrestrial habitats, however, most studies focus

61 on soil and the rhizosphere (Arcate et al., 2006; Esmaeili Taheri et al., 2017; Sapp et al.,

62 2019; Fiore-Donno \& Bonkowski, 2021). Recently, Jauss et al. (2020b) characterised

63 oomycete diversity and community composition in tree canopies, which are huge

64 ecosystems containing heterogeneous microhabitats and a large proportion of undescribed

65 diversity (Nadkarni, 2001). Albeit the same oomycetes were present on the ground and in

66 the canopy, communities inhabiting canopy habitats were significantly distinct from soil and

67 leaf litter communities in their abundances. The authors concluded that oomycete diversity in

68 forest ecosystems is shaped by deterministic microhabitat filtering, while a study by Jauss et

69 al. (2020a) could determine air dispersal and convective transport to be the stochastic

70 supplier and distributor of oomycetes among microhabitats and strata. However, the former

71 study only analysed one time point, while the latter study dealing with air samples could

72 show a strong temporal variability in community composition. Accordingly, seasonal

73 variability has been shown to influence protistan communities, to some extent, in several

74 studies (Nolte et al., 2010; Fiore-Donno et al., 2019; Fournier et al., 2020; Walden et al.,

75 2021). For cercozoan communities, Walden et al. (2021) could show annually reoccurring

76 succession patterns in the phyllosphere. This implied not only spatially, but also seasonally

77 structured cercozoan communities in tree canopies, although this was not reflected on a 
78 functional scale. If seasonal variation is also reflected in the functional diversity of oomycetes

79 in forest ecosystems, however, remains elusive.

80 Accordingly, we supplemented functional traits and investigated the seasonal stability of

81 oomycete community composition in forest floors and tree canopies over a period of two

82 years. Our study tackles two hypotheses: (1) Oomycete communities vary not only in their

83 spatial distribution, but also in their seasonal composition, and (2) the deterministic

84 processes leading to differences in community composition between canopy and ground

85 habitats also shape the functional diversity and functional distribution among microhabitats.

86 2 MATERIAL AND METHODS

$87 \quad 2.1$ Sampling, DNA extraction and sequencing

88 Microhabitat samples were collected in two seasons over a period of two years, i.e. autumn

89 (October) 2017 and 2018 and spring (May) 2018 and 2019 in cooperation with the Leipzig

90 Canopy Crane (LCC) Facility in a floodplain forest in Leipzig, Germany (51.3657 N, 12.3094

91 E). Samples were obtained and processed as described in Jauss et al. (2020b). Briefly,

92 seven microbial microhabitat compartments related to tree surface were sampled in the

93 canopy at 20-30m height: Fresh leaves, dead wood, bark, arboreal soil and three cryptogam

94 epiphytes (lichen and two moss genera, Hypnum and Orthotrichum). In addition, two ground

95 samples (soil and leaf litter) were sampled. All microhabitat samples were taken with four

96 replicates, from three tree species with three replicates each. DNA extraction was performed

97 with the DNeasy PowerSoil kit (QIAGEN, Hilden, Germany) according to the manufacturer's

98 instruction. This procedure was performed on four sampling dates: October 2017 (Jauss et

99 al., 2020b), May 2018, October 2018 and May 2019 (this study). Oomycete-specific PCRs

100 and sequencing were performed as described in Jauss et al. (2020b) with tagged primers 
101 designed by Fiore-Donno \& Bonkowski (2021); the used primer tag combinations are 102 provided in Supplementary Table 1.

\section{$103 \quad 2.2$ Sequence processing}

104 Sequence processing and bioinformatics analyses followed the pipeline described in Jauss 105 et al. (2020b). Briefly, raw reads were merged using VSEARCH v2.10.3 (Rognes et al., 106 2016) and demultiplexed with cutadapt v1.18 (M. Martin, 2011). Primer and tag sequences 107 were trimmed and concatenated sequencing runs were then clustered into operational 108 taxonomic units (OTUs) using Swarm v2.2.2 (Mahé et al., 2015). Chimeras were de novo 109 detected using VSEARCH. OTUs were removed from the final OTU table if they were 110 flagged as chimeric, showed a quality value of less than 0.0002 , were shorter than $150 \mathrm{bp}$, or 111 were represented by less than $0.005 \%$ of all reads (i.e. 368 reads). OTUs were first 112 taxonomically assigned by using BLAST+ v2.9.0 (Camacho et al., 2009) with default 113 parameters against the non-redundant NCBI Nucleotide database (as of June 2019) and 114 removed if the best hit in terms of bitscore was a non-oomycete sequence. Finer taxonomic 115 assignment was performed with VSEARCH on a custom oomycete ITS1 database (Jauss et 116 al., 2020b). The annotation was refined by assigning the species name of the best 117 VSEARCH hit to the corresponding OTU if the pairwise identity was over $95 \%$, OTUs with 118 lower percentages were assigned higher taxonomic levels. Functional annotation was 119 performed on genus level with a custom python script, based on the oomycete functional 120 database published by Fiore-Donno \& Bonkowski (2021). Samples with low sequencing 121 depth were removed by loading the final OTU table into QIIME 2 v2018.11 (Bolyen et al., 122 2019) and retaining at least five samples per microhabitat and 15 samples per tree species 123 per sampling date, i.e. samples with at least 1172 reads. Additionally, the oomycete OTU 124 abundance matrix of air samples from Jauss et al. (2020a) was used for a comparison 125 between tree related microhabitats and the surrounding air from spring 2019, as these 126 samples were taken simultaneously. 


\section{$127 \quad 2.3$ Statistical analyses}

128 All statistical analyses were conducted in R v3.5.3 (R Core Team, 2019). Alpha diversity

129 indices were calculated for each sample using the diversity function in the vegan package

130 (Oksanen et al., 2019). Non-metric multidimensional scaling was performed on the Bray-

131 Curtis dissimilarity matrix of the log transformed relative abundances (functions vegdist and

132 metaMDS in the vegan package, respectively), the same matrix was used for a 133 permutational multivariate analysis of variance (permANOVA) with the adonis function.

134 Partitioning and visualisation of relative abundances between canopy, soil and leaf litter was 135 performed with the ggtern package (Hamilton \& Ferry, 2018). Determination of significantly 136 differentially abundant OTUs was performed with the DESeq2 package (Love et al., 2014). 137 All figures were plotted with the ggplot2 package (Wickham, 2016).

\section{RESULTS}

\subsection{Taxonomic and functional annotation}

140 We obtained 375 OTUs from $4,262,960$ sequences. 77 OTUs (= $20.5 \%$ of all OTUs) showed

141 a sequence similarity of less than $70 \%$ to any known reference sequence. Plotting the 142 sequence similarity against reference sequences revealed similar patterns as previously 143 described by Jauss et al. (2020b), i.e., many OTUs showed a similarity of $97-100 \%$ to known 144 reference sequences, while additional peaks at $\sim 75 \%$ and $\sim 85 \%$ may indicate hitherto 145 undescribed oomycete lineages (Supplementary Figure 1).

146 Peronosporales and Pythiales dominated all microhabitats at all sampling events 147 (Supplementary Figure 2). Distribution of functional groups was relatively constant for all four 148 sampling events (Figure 1). Based on OTU presence/absence, the pattern was nearly 149 identical for all microhabitats (Figure 1A-D). Approximately $20 \%$ of all OTUs occupied a 150 hemibiotrophic lifestyle, $30 \%$ were determined to be obligate biotrophic, only few OTUs 
151 belonged to saprotrophic species and the lifestyle of the remaining $50 \%$ of OTUs could not

152 be determined, mainly due to low sequence similarities to reference sequences. However,

153 when taking abundances of OTUs into account, the pattern clearly shifted. OTUs assigned to

154 obligate biotrophic species dominated canopy habitats, while ground habitats were more

155 dominated by hemibiotrophic species (Figure 1E-H).

156 Comparing the data from Spring 2019 (Figure 1D,H) with air samples previously published

157 by Jauss et al. (2020a) (Figure 2) revealed that the air surrounding canopy and ground 158 habitats was dominated by obligate biotrophic OTUs, irrespective of incidence or 159 abundance.

\subsection{Abundance partitioning}

\section{3.2.1 Partitioning between Canopy, Soil and Leaf Litter}

162

163

164

165

166

167

168

169

170

171

172

173

174
To further determine the distribution of functional groups together with the taxonomic annotation, the relative abundances of each OTU were partitioned for canopy, soil and leaf litter samples (Figure 3). Again, OTUs assigned to obligate biotrophic species dominated canopy samples, while hemibiotrophic species were more evenly distributed or more abundant in leaf litter and soil habitats. Albuginales were almost exclusively present in canopy samples, Peronosporales dominated canopy and leaf litter samples, while Pythiales showed a rather even distribution.

The relative abundances of the latter two orders were further partitioned into the four sampling events (Supplementary Figure 3). Abundances of Pythiales were rather homogenous and consistent throughout the seasons, while Peronosporales abundances were more shifted to the canopy region in spring samples. In Autumn 2017, OTUs assigned to the Peronosporales were almost exclusively present in canopy and leaf litter samples, while the distribution in Autumn 2018 was more homogenous. 


\section{3.2.2 Differential Abundance Analysis}

176 To determine which OTU abundances were significantly different between the two strata

177 ground and canopy as well as the two sampling seasons spring and autumn, a differential

178 abundance analysis was carried out (Figure 4, Supplementary Figure 4). Within the

179 Peronosporales, this revealed the genera Peronospora and Hyaloperonospora (obligate

180 biotrophic genera) to be the dominant taxa in canopy samples, while Phytophthora

181 (hemibiotrophic) species were significantly differentially abundant in ground samples (Figure

182 4). For the seasonal effect, more Peronospora species were differentially abundant in spring

183 samples compared to autumn samples (Supplementary Figure 4). Within the Pythiales, the

184 genera Pythium (hemibiotrophic) and Globisporangium (obligate biotrophic) were

185 significantly differentially abundant in ground samples. Most Pythiales, however, could not

186 be determined due to the low sequence similarity to reference sequences.

\section{$187 \quad 3.3$ Alpha and beta diversity}

188 Despite OTU richness being quite variable among microhabitats, Shannon diversity as well 189 as evenness were high and did not differ between the samplings (Supplementary Figure 5).

190 Beta diversity analyses revealed similar patterns for all seasons as well: the NMDS plot

191 (Figure 5) showed a large overlap of canopy inhabiting communities, which in turn did not

192 overlap with leaf litter and soil communities. This indicated distinct communities inhabiting

193 canopy and ground habitats, respectively, a pattern recurring in all samplings.

194 Variation in community composition was twice as high among microhabitats $\left(\mathrm{R}^{2}=0.20\right)$ than 195 between canopy and ground $\left(R^{2}=0.11\right)$ or sampling dates $\left(R^{2}=0.10\right)$. Tree species $\left(R^{2}=0.05\right)$

196 and season $\left(R^{2}=0.04\right)$ explained only a minor fraction of beta diversity (permANOVA, Table 197 1). 


\section{DISCUSSION}

199 The most striking pattern of oomycete community composition is the distribution of obligate

200 biotrophic and hemibiotrophic species, with the former dominating canopy habitats and the

201 latter predominantly found in ground habitats (Figure 1). In a previous study, Jauss et al.

202 (2020b) proposed increasing functional diversity instead of increasing species richness with

203 increasing habitat diversity, as most OTUs were shared between all habitats irrespective of

204 specific strata or tree species. Here we supplemented functional traits of the detected OTUs,

205 which revealed that the observed diversity is driven by the lifestyle of the oomycetes.

206 Species occupying a hemibiotrophic lifestyle dominated the two ground habitats soil and leaf

207 litter. Hemibiotrophy is characterised by an initial biotrophic phase, which turns into a

208 necrotrophic phase (Fawke et al., 2015; Pandaranayaka et al., 2019). Oomycetes dwelling

209 the ground habitats are thus capable of feeding on the dead organic matter in the soil, leaf

210 litter and deadwood samples. Deadwood on the forest floor has already been shown to

211 harbour hemibiotrophic oomycetes (Kwaśna et al. 2017a; 2017b). In the canopy, however,

212 deadwood harbours only little hemibiotrophic species, as they are dominated by obligate

213 biotrophic species, like the other canopy habitats. The reason for this might be the high

214 number of obligate biotrophs in the other surrounding canopy habitats as well as in the air

215 (Figure 2). These samples might be overwhelmed by the passive influx of biotrophic species,

216 which are capable of surviving in the other, living, habitats, which would be an interplay

217 between stochastic and deterministic processes for community assembly.

218 Recent molecular studies analysing oomycete diversity determined similar patterns as

219 reflected in our study, i.e. soil habitats are dominated by hemibiotrophic species, mostly

220 members of the Pythiales (Sapkota \& Nicolaisen, 2015; Riit et al., 2016; Fiore-Donno \&

221 Bonkowski, 2021). Species of the genus Pythium were significantly differentially abundant in

222 our ground habitats. Habitats in the canopy, however, were dominated by the obligate

223 biotrophic genera Peronospora and Hyaloperonospora (Figure 4). Tree canopies have only

224 recently been subject to studies on microbial diversity (Jauss et al., 2020a, 2020b; Walden et 
225 al., 2021; Herrmann et al., 2021), indicating tree canopies to be a hitherto neglected 226 reservoir for parasitic microorganisms. Species of the genus Hyaloperonospora are known to 227 be highly host-specific, infecting plant species of Brassicaceae and closely related families 228 (Lee et al., 2017 and references therein). However, none of our sampled trees and 229 microhabitats belong to the Brassicaceae or the order Brassicales. Yet, we observed a high 230 number of reads and OTUs assigned to the genus Hyaloperonospora in the microhabitat 231 samples in the canopy as well as in the air samples in both strata, while their number in 232 ground microhabitats is significantly depleted (Figure 4). This indicates a non-random 233 distribution of Hyaloperonospora species, as the air as a distribution mechanism should lead 234 to a more or less equal distribution in canopy and ground habitats. Here, they should not be 235 able to survive due to their high host specificity. But the domination in canopy samples 236 implies a capability of survival on hosts they are not specialised on. Thus, we tentatively 237 propose an even less strict host dependency for the genus Hyaloperonospora than already 238 suggested (Yerkes \& Shaw, 1959; McMeekin, 1960; Dickinson \& Greenhalgh, 1977).

239 The significant differential abundance in the canopy of several undetermined OTUs that can 240 only be assigned to the family Pythiaceae (Figure 4) indicates hitherto undescribed lineages, 241 specialised on the survival in the canopy. Members of the Pythiaceae can occupy all 242 lifestyles, from saprotrophy over hemibiotrophy to obligate biotrophy (Fawke et al., 2015; 243 Marano et al., 2016; Fiore-Donno \& Bonkowski, 2021). If the OTUs in the canopy would 244 show an obligate biotrophic lifestyle, it would be in line with observations of the other 245 lineages in the canopy (Figure 1). Yet, the sequence similarity of these OTUs amounts to 246 only ca $80-85 \%$ to any reference sequence, thus we only tentatively draw conclusions about 247 their lifestyle.

248 A common pattern in microbial community ecology studies is a high seasonal variability 249 (Nolte et al., 2010; Fiore-Donno et al., 2019; Fournier et al., 2020; Walden et al., 2021). 250 Oomycete community compositions were in fact slightly, yet significantly distinct for every 251 sampling and correspondingly for every season (Table 1). This pattern is in line with 252 hypotheses proposed by Jauss et al. (2020a), that seasonal variation in air samples drives 
253 the community composition in forest ecosystems. The environment, however, then selects

254 the species most adapted to the microhabitat, leading to overall similar community patterns

255 and microhabitat differences for every season (Figure 5). The seasonal changes in

256 microhabitat properties (e.g. temperature, moisture or habitat structure) thus affect all

257 habitats and communities equally. The season itself explained less variance in community

258 composition than the sampling dates (i.e., Autumn 2017 vs. Autumn 2018 etc.; Table 1),

259 suggesting that annual changes do not lead to similar community structures within

260 microhabitats in each season as an annual cycle per se, but rather indicate a high temporal

261 variability while preserving spatial diversity. Fournier et al. (2020) observed similar patterns,

262 concluding deterministic niche-based processes in microbial forest soil community assembly.

263 Implications are that ecosystem functioning of oomycete communities is not mainly affected

264 by seasonal fluctuations, but rather by microhabitat identity and, correspondingly, responses

265 of lifestyle to microhabitat filtering (Fiore-Donno \& Bonkowski, 2021).

266 Conclusions

267 Both our hypotheses were confirmed in this study: Oomycetes show not only a spatial, but,

268 to a lesser extent, also a temporal variation in their communities. Within the temporal

269 variation however, the spatial variation is preserved, leading to overall similar community

270 patterns for every sampling date. Further, these deterministic processes also shape their

271 functional diversity in forest ecosystems. Our results indicate that tree canopies not only

272 offer numerous distinct habitats to microorganisms, but also serve as a reservoir for parasitic

273 species. Spatial diversity and correspondingly functional diversity drive the oomycete

274 community to a greater extent than temporal diversity. Thus, our findings contribute to future

275 studies on oomycete ecosystem functioning. 


\section{Funding}

277 This work was supported by the Priority Program SPP 1991: Taxon-omics - New

278 Approaches for Discovering and Naming Biodiversity of the German Research Foundation

279 (DFG) with funding to MB (1907/19-1) and MS (Schl 229/20-1). We acknowledge support

280 from the Leipzig University Library for open access publishing.

281 Acknowledgements

282 The authors would like to thank Rolf Engelmann for his assistance with the field work by

283 operating the canopy crane, as well as the Leipzig Canopy Crane Platform of the German

284 Centre for Integrative Biodiversity Research (iDiv) for providing the site access and allowing

285 us to sample the trees from their field trial.

286 Conflict of Interest

287 None declared.

288 References

289

290

291

292

293

294

295

296

297

298

299

300

301

302

303
Adl, S. M., Bass, D., Lane, C. E., Lukeš, J., Schoch, C. L., Smirnov, A., Agatha, S., Berney, C., Brown, M. W., Burki, F., Cárdenas, P., Čepička, I., Chistyakova, L., del Campo, J., Dunthorn, M., Edvardsen, B., Eglit, Y., Guillou, L., Hampl, V., ... Zhang, Q. (2019). Revisions to the Classification, Nomenclature, and Diversity of Eukaryotes. Journal of Eukaryotic Microbiology, 66, 4-119. https://doi.org/10.1111/jeu.12691

Arcate, J. M., Karp, M. A., \& Nelson, E. B. (2006). Diversity of Peronosporomycete (oomycete) communities associated with the rhizosphere of different plant species. Microbial Ecology, 51(1), 36-50. https://doi.org/10.1007/s00248-005-0187-y

Bolyen, E., Rideout, J. R., Dillon, M. R., Bokulich, N. A., Abnet, C. C., Al-Ghalith, G. A., Alexander, H., Alm, E. J., Arumugam, M., Asnicar, F., Bai, Y., Bisanz, J. E., Bittinger, K., Brejnrod, A., Brislawn, C. J., Brown, C. T., Callahan, B. J., Caraballo-Rodríguez, A. M., Chase, J., ... Caporaso, J. G. (2019). Reproducible, interactive, scalable and extensible microbiome data science using QIIME 2. Nature Biotechnology, 37(8), 852857. https://doi.org/10.1038/s41587-019-0209-9

Camacho, C., Coulouris, G., Avagyan, V., Ma, N., Papadopoulos, J., Bealer, K., \& Madden, 
304

305

306

307

308

309

310

311

312

313

314

315

316

317

318

319

320

321

322

323

324

325

326

327

328

329

330

331

332

333

334

335

336

337

338

339

340

341

342

343

344

345

346

347

348

349

350

351

352

353

354

355

356

357

358
T. L. (2009). BLAST+: Architecture and applications. BMC Bioinformatics, 10. https://doi.org/10.1186/1471-2105-10-421

Dickinson, C. H., \& Greenhalgh, J. R. (1977). Host range and taxonomy of Peronospora on crucifers. Transactions of the British Mycological Society, 69(1), 111-116. https://doi.org/10.1016/s0007-1536(77)80121-6

Esmaeili Taheri, A., Chatterton, S., Gossen, B. D., \& McLaren, D. L. (2017). Metagenomic analysis of oomycete communities from the rhizosphere of field pea on the Canadian prairies. Canadian Journal of Microbiology, 63(9), 758-768. https://doi.org/10.1139/cjm2017-0099

Fawke, S., Doumane, M., \& Schornack, S. (2015). Oomycete Interactions with Plants: Infection Strategies and Resistance Principles. Microbiology and Molecular Biology Reviews, 79(3), 263-280. https://doi.org/10.1128/mmbr.00010-15

Fiore-Donno, A. M., \& Bonkowski, M. (2021). Different community compositions between obligate and facultative oomycete plant parasites in a landscape-scale metabarcoding survey. Biology and Fertility of Soils, 57(2), 245-256. https://doi.org/10.1007/s00374020-01519-z

Fiore-Donno, A. M., Richter-Heitmann, T., Degrune, F., Dumack, K., Regan, K. M., Marhan, S., Boeddinghaus, R. S., Rillig, M. C., Friedrich, M. W., Kandeler, E., \& Bonkowski, M. (2019). Functional traits and spatio-temporal structure of a major group of soil protists (rhizaria: Cercozoa) in a temperate grassland. Frontiers in Microbiology, 10(JUN). https://doi.org/10.3389/fmicb.2019.01332

Fournier, B., Samaritani, E., Frey, B., Seppey, C. V. W., Lara, E., Heger, T. J., \& Mitchell, E. A. D. (2020). Higher spatial than seasonal variation in floodplain soil eukaryotic microbial communities. Soil Biology and Biochemistry, 147, 107842. https://doi.org/10.1016/j.soilbio.2020.107842

Hamilton, N. E., \& Ferry, M. (2018). Ggtern: Ternary diagrams using ggplot2. Journal of Statistical Software, 87(1), 1-17. https://doi.org/10.18637/jss.v087.c03

Herrmann, M., Geesink, P., Richter, R., \& Küsel, K. (2021). Canopy Position Has a Stronger Effect than Tree Species Identity on Phyllosphere Bacterial Diversity in a Floodplain Hardwood Forest. Microbial Ecology, 81(1), 157-168. https://doi.org/10.1007/s00248020-01565-y

Jauss, R.-T., Nowack, A., Walden, S., Wolf, R., Schaffer, S., Schellbach, B., Bonkowski, M., \& Schlegel, M. (2020a). To the canopy and beyond: Air samples reveal wind dispersal as a driver of ubiquitous protistan pathogen assembly in tree canopies. BioRxiv, 2020.11.30.405688. https://doi.org/10.1101/2020.11.30.405688

Jauss, R.-T., Walden, S., Fiore-Donno, A. M., Dumack, K., Schaffer, S., Wolf, R., Schlegel, M., \& Bonkowski, M. (2020b). From forest soil to the canopy: Increased habitat diversity does not increase species richness of Cercozoa and Oomycota in tree canopies. Frontiers in Microbiology, 11, 3364. https://doi.org/10.3389/FMICB.2020.592189

Kamoun, S., Furzer, O., Jones, J. D. G., Judelson, H. S., Ali, G. S., Dalio, R. J. D., Roy, S. G., Schena, L., Zambounis, A., Panabières, F., Cahill, D., Ruocco, M., Figueiredo, A., Chen, X. R., Hulvey, J., Stam, R., Lamour, K., Gijzen, M., Tyler, B. M., ... Govers, F. (2015). The Top 10 oomycete pathogens in molecular plant pathology. Molecular Plant Pathology, 16(4), 413-434. https://doi.org/10.1111/mpp.12190

Krsteska, V., Dimeska, V., Stojkov, S., \& Stojanoski, P. (2014). Peronospora Tabacina A. The causing agent of blue mold disease on Tobacco. Bulgarian Journal of Agricultural Science, 21(1), 126-133. http://eprints.uklo.edu.mk/312/

Kwaśna, H., Mazur, A., Kuźmiński, R., Jaszczak, R., Turski, M., Behnke-Borowczyk, J., Adamowicz, K., \& Łakomy, P. (2017a). Abundance and diversity of wood-decay fungi in managed and unmanaged stands in a Scots pine forest in western Poland. Forest Ecology and Management, 400, 438-446. https://doi.org/10.1016/j.foreco.2017.04.023

Kwaśna, H., Mazur, A., Łabędzki, A., Kuźmiński, R., \& Łakomy, P. (2017b). Communities of fungi in decomposed wood of oak and pine. Forest Research Papers, 77(3), 261-275. https://doi.org/10.1515/frp-2016-0028

Lee, J. S., Lee, H. B., Shin, H. D., \& Choi, Y. J. (2017). Diversity, phylogeny, and host- 
specialization of Hyaloperonospora species in Korea. Mycobiology, 45(3), 139-149. https://doi.org/10.5941/MYCO.2017.45.3.139

Lewis, D. H. (1973). Concepts in fungal nutrition and the origin of biotrophy. Biological Reviews, 48(2), 261-277. https://doi.org/10.1111/j.1469-185x.1973.tb00982.x

Love, M. I., Huber, W., \& Anders, S. (2014). Moderated estimation of fold change and dispersion for RNA-seq data with DESeq2. Genome Biology, 15(12), 550. https://doi.org/10.1186/s13059-014-0550-8

Mahé, F., Rognes, T., Quince, C., de Vargas, C., \& Dunthorn, M. (2015). Swarmv2: Highlyscalable and high-resolution amplicon clustering. PeerJ, 2015(12). https://doi.org/10.7717/peerj.1420

Marano, A. V., Jesus, A. L., de Souza, J. I., Jerônimo, G. H., Gonçalves, D. R., Boro, M. C., Rocha, S. C. O., \& Pires-Zottarelli, C. L. A. (2016). Ecological roles of saprotrophic Peronosporales (Oomycetes, Straminipila) in natural environments. Fungal Ecology, 19, 77-88. https://doi.org/10.1016/j.funeco.2015.06.003

Marano, A. V, Jesus, A. L., Pires-Zottarelli, C. L. A., James, T. Y., Gleason, F. H., \& de Souza, J. I. (2014). Phylogenetic relationships of Pythiales and Peronosporales (Oomycetes, Straminipila) within the "peronosporalean galaxy".." In K. Jones, EBG and Hyde, KD and Pang (Ed.), Freshwater fungi and fungal-like organisms (pp. 177-199). WALTER DE GRUYTER https://doi.org/https://doi.org/10.1515/9783110333480.177

Martin, F., Kohler, A., Murat, C., Veneault-Fourrey, C., \& Hibbett, D. S. (2016). Unearthing the roots of ectomycorrhizal symbioses. In Nature Reviews Microbiology (Vol. 14, Issue 12, pp. 760-773). Nature Publishing Group. https://doi.org/10.1038/nrmicro.2016.149

Martin, M. (2011). Cutadapt removes adapter sequences from high-throughput sequencing reads. EMBnet.Journal, 17(1), 10. https://doi.org/10.14806/ej.17.1.200

McMeekin, D. (1960). The role of the oospores of Peronospora parasitica in downy mildew $\begin{array}{llll}\text { of } \quad \text { crucifera. } & \text { Phytopathology, } & \text { 93-97 } & \text { pp. }\end{array}$ https://www.cabdirect.org/cabdirect/abstract/19601101892

Mizubuti, E. S. ., \& Fry, W. E. (2006). Potato late blight. In The Epidemiology of Plant Diseases (pp. 445-471). Kluwer Academic Publishers. https://doi.org/10.1007/1-40204581-6_17

Nadkarni, N. M. (2001). Enhancement of forest canopy research, education, and conservation in the new millennium. In K. E. Linsenmair, A. J. Davis, B. Fiala, \& M. R. Speight (Eds.), Tropical Forest Canopies: Ecology and Management (pp. 361-367). Springer. https://doi.org/10.1007/978-94-017-3606-0_29

Nolte, V., Pandey, R. V., Jost, S., Medinger, R., Ottenwälder, B., Boenigk, J., \& Schlötterer, C. (2010). Contrasting seasonal niche separation between rare and abundant taxa conceals the extent of protist diversity. Molecular Ecology, 19(14), 2908-2915. https://doi.org/10.1111/j.1365-294X.2010.04669.x

Oksanen, J., Blanchet, F. G., Friendly, M., Kindt, R., Legendre, P., McGlinn, D., Minchin, P. R., O'Hara, R. B., Simpson, G. L., Solymos, P., Stevens, M. H. H., Szoecs, E., \& Wagner, H. (2019). vegan: Community Ecology Package. https://cran.rproject.org/package $=$ vegan

Pandaranayaka, E. P. J., Frenkel, O., Elad, Y., Prusky, D., \& Harel, A. (2019). Network analysis exposes core functions in major lifestyles of fungal and oomycete plant pathogens. BMC Genomics, 20(1), 1-15. https://doi.org/10.1186/s12864-019-6409-3

Parkunan, V., Gitaitis, R. D., Dutta, B., Langston, D. B., \& Ji, P. (2013). An Epidemic of Downy Mildew caused by Peronospora destructor on Vidalia Sweet Onions in Georgia in 2012. Plant Health Progress, 14(1), 54. https://doi.org/10.1094/php-2013-0328-01-br

R Core Team. (2019). R: A Language and Environment for Statistical Computing. https://www.r-project.org/

Riit, T., Tedersoo, L., Drenkhan, R., Runno-Paurson, E., Kokko, H., \& Anslan, S. (2016). Oomycete-specific ITS primers for identification and metabarcoding. MycoKeys, 14, 1730. https://doi.org/10.3897/mycokeys.14.9244

Rizzo, D. M., \& Garbelotto, M. (2003). Sudden Oak Death: Endangering California and 
414

415

416

417

418

419

420

421

422

423

424

425

426

427

428

429

430

431

432

433

434

435

436

437

438

439

440

441

442

443

444

445

446

447

448

449

450

451

452

453

454

455

456

457

458

Oregon Forest Ecosystems. Frontiers in Ecology and the Environment, 1(4), 197. https://doi.org/10.2307/3868064

Rizzo, D. M., Garbelotto, M., \& Hansen, E. M. (2005). Phytophthora ramorum: Integrative Research and Management of an Emerging Pathogen in California and Oregon Forests. Annual Review of Phytopathology, 43(1), 309-335. https://doi.org/10.1146/annurev.phyto.42.040803.140418

Robideau, G. P., De Cock, A. W. A. M., Coffey, M. D., Voglmayr, H., Brouwer, H., Bala, K., Chitty, D. W., Désaulniers, N., Eggertson, Q. A., Gachon, C. M. M., Hu, C. H., Küpper, F. C., Rintoul, T. L., Sarhan, E., Verstappen, E. C. P., Zhang, Y., Bonants, P. J. M., Ristaino, J. B., \& André Lévesque, C. (2011). DNA barcoding of oomycetes with cytochrome c oxidase subunit I and internal transcribed spacer. Molecular Ecology Resources, 11(6), 1002-1011. https://doi.org/10.1111/j.1755-0998.2011.03041.x

Rognes, T., Flouri, T., Nichols, B., Quince, C., \& Mahé, F. (2016). VSEARCH: A versatile open source tool for metagenomics. PeerJ, 2016(10). https://doi.org/10.7717/peerj.2584

Sapkota, R., \& Nicolaisen, M. (2015). An improved high throughput sequencing method for studying oomycete communities. Journal of Microbiological Methods, 110, 33-39. https://doi.org/10.1016/j.mimet.2015.01.013

Sapp, M., Tyborski, N., Linstädter, A., López Sánchez, A., Mansfeldt, T., Waldhoff, G., Bareth, G., Bonkowski, M., \& Rose, L. E. (2019). Site-specific distribution of oak rhizosphere-associated oomycetes revealed by cytochrome $c$ oxidase subunit II metabarcoding. Ecology and Evolution, 9(18), 10567-10581. https://doi.org/10.1002/ece3.5577

Singer, D., Lara, E., Steciow, M. M., Seppey, C. V. W., Paredes, N., Pillonel, A., Oszako, T., \& Belbahri, L. (2016). High-throughput sequencing reveals diverse oomycete communities in oligotrophic peat bog micro-habitat. Fungal Ecology, 23, 42-47. https://doi.org/10.1016/j.funeco.2016.05.009

Spanu, P., \& Kämper, J. (2010). Genomics of biotrophy in fungi and oomycetes-emerging patterns. In Current Opinion in Plant Biology (Vol. 13, Issue 4, pp. 409-414). Elsevier Current Trends. https://doi.org/10.1016/j.pbi.2010.03.004

Spanu, P., \& Panstruga, R. (2017). Editorial: Biotrophic plant-microbe interactions. In Frontiers in Plant Science (Vol. 8, p. 192). Frontiers Research Foundation. https://doi.org/10.3389/fpls.2017.00192

Thines, M., \& Kamoun, S. (2010). Oomycete-plant coevolution: Recent advances and future prospects. In Current Opinion in Plant Biology (Vol. 13, Issue 4, pp. 427-433). Curr Opin Plant Biol. https://doi.org/10.1016/j.pbi.2010.04.001

Walden, S., Jauss, R.-T., Feng, K., Fiore-Donno, A. M., Schaffer, S., Wolf, R., Schlegel, M., \& Bonkowski, M. (2021). On the phenology of protists: Recurrent patterns reveal seasonal variation of protistan (Rhizaria: Cercozoa, Endomyxa) communities in tree canopies. BioRxiv, 2021.02.15.431229. https://doi.org/10.1101/2021.02.15.431229

Wickham, H. (2016). ggplot2: Elegant Graphics for Data Analysis. Springer-Verlag New York. https://ggplot2.tidyverse.org

Yerkes, W. D., \& Shaw, C. G. (1959). Taxonomy of the Peronospora species on Cruciferae and Chenopodiaceae. Phytopathology, 49(8), 499-507 pp. https://www.cabdirect.org/cabdirect/abstract/19601100570 


\section{Data Accessibility}

460 Raw sequence data have been submitted to the European Nucleotide Archive (ENA)

461 database under the Bioproject number PRJEB37525, with accession numbers ERS4399744, $462 \quad$ ERS5649966 and ERS5649967.

463 All figures, codes and detailed bioinformatic/statistical methods used in this study are 464 available at https://github.com/RJauss/ParasitesParadise.

465 Author contributions

466 MB and MS conceived the study. RW and StS designed the sampling and DNA extraction.

467 AMF-D contributed the primers and functional annotation of oomycetes. SW and R-TJ

468 conducted the sampling, DNA extraction and PCRs. KF assisted DNA extraction and PCRs.

469 RT-J performed the bioinformatic and statistical analyses and drafted the manuscript. All

470 authors contributed to and approved the final version.

471 Tables

Table 1: Results of permutational multivariate analysis of variance (permANOVA) from the provides the two factors Autumn and Spring, while Sampling Date corresponds to the specific time points of sampling, i.e. Autumn 2017, Spring 2018 etc.

\begin{tabular}{|c|c|c|c|c|c|}
\hline & $D f$ & SumsOfSqs & Fvalue & $R 2$ & $p$ \\
\hline Tree Species & 2 & 5.18 & 7.95 & 0.05 & 0.001 \\
\hline Microhabitat & 8 & 20.45 & 9.12 & 0.20 & 0.001 \\
\hline Stratum & 1 & 10.78 & 35.20 & 0.11 & 0.001 \\
\hline Season & 1 & 4.00 & 12.15 & 0.04 & 0.001 \\
\hline Sampling Date & 3 & 10.32 & 11.10 & 0.10 & 0.001 \\
\hline
\end{tabular}




\section{Figures}

478

479

480

481

482

483

484

485

486

487

488

489

490

491

492

493

494

495

496

497

498

499

500

501

502

503

504

505

506

507

508

509

510

511

512

513

514

Figure 1: Functional annotation of oomycete OTUs in canopy and ground habitats. (A-D) Distribution of functional groups based on OTU presence/absence, i.e. the proportion of OTUs per Lifestyle. (E-H) Distribution of functional groups when taking abundances into account. $A=$ Arboreal Soil, $\mathrm{B}=$ Bark, $\mathrm{D}=$ Deadwood, $\mathrm{F}=$ Fresh Leaves, $\mathrm{H}=$ Hypnum, $\mathrm{Li}=$ Lichen, $\mathrm{O}=$ Orthotrichum, $\mathrm{S}=$ Soil, LL = Leaf Litter

Figure 2: Functional annotation of oomycete OTUs from Spring 2019. Microhabitat samples based on OTU presence/absence (A) and OTU abundances (C) compared to air samples based on OTU presence/absence (B) and OTU abundances (D). For microhabitat abbreviations, see Figure 1.

Figure 3: Ternary plot partitioning the relative abundances of OTUs between canopy, soil and leaf litter. Each dot represents one OTU, sorted by taxonomic order and coloured by lifestyle. Incertae sedis comprises families and genera not associated with any order, e.g. Lagenaceae or Paralagenidium. The order Undetermined represents OTUs with sequence similarities of less than $70 \%$ to any reference sequence.

Figure 4: Differential abundance analysis between the two strata canopy (top panels) and ground (bottom panels) sorted by taxonomic order. Each dot represents one significantly differentially abundant OTU grouped by genus. Y-axis (log2FoldChange) gives the measurement of the differential abundance.

Figure 5: Non-metric multidimensional scaling (NMDS) ordination of Bray-Curtis dissimilarity matrices for canopy and ground microhabitats. Canopy microhabitat communities show a large overlap along all sampling events. Ground habitat communities are strongly separated, indicating unique exclusive communities compared to the canopy region, irrespective of the sampling season.

\section{Supplementary Figures}

Supplementary Figure 1: Sequence similarity of reads (top) and OTUs (bottom) per sampling event to published reference sequences. $20.5 \%$ of all OTUs, corresponding to $3 \%$ of all reads, had a similarity of less than $70 \%$ to any known reference sequence (not shown).

Supplementary Figure 2: Taxonomic assignment of OTUs per sampling and microhabitat. Black line separates canopy and ground habitats. Distribution of taxonomic groups was similar for every sampling, i.e. Pythiales and Peronosporales dominating all samples.

Supplementary Figure 3: Ternary plot partitioning the relative abundances of Peronosporales and Pythiales per sampling event. Each dot represents one OTU.

Supplementary Figure 4: Differential abundance analysis between the two seasons spring (top panels) and autumn (bottom panels) sorted by taxonomic order. Each dot represents one significantly differentially abundant OTU grouped by genus. Y-axis (log2FoldChange) gives the measurement of the differential abundance.

Supplementary Figure 5: Boxplot of alpha diversity indices for microhabitat communities per sampling. Outliers are given by dots. Observed patterns show no strong variability over the four sampling events. 
bioRxiv preprint doi: https://doi.org/10.1101/2021.02.17.431613; this version posted February 17, 2021. The copyright holder for this preprint (which was not certified by peer review) is the author/funder, who has granted bioRxiv a license to display the preprint in perpetuity. It is made available under aCC-BY-NC-ND 4.0 International license.

\section{Supplementary Tables}

516 Supplementary Table 1: Primer tags used in this study. Given are the sample ID, forward and 517 (reverse complemented) reverse tag and the ENA sequencing run ID. 


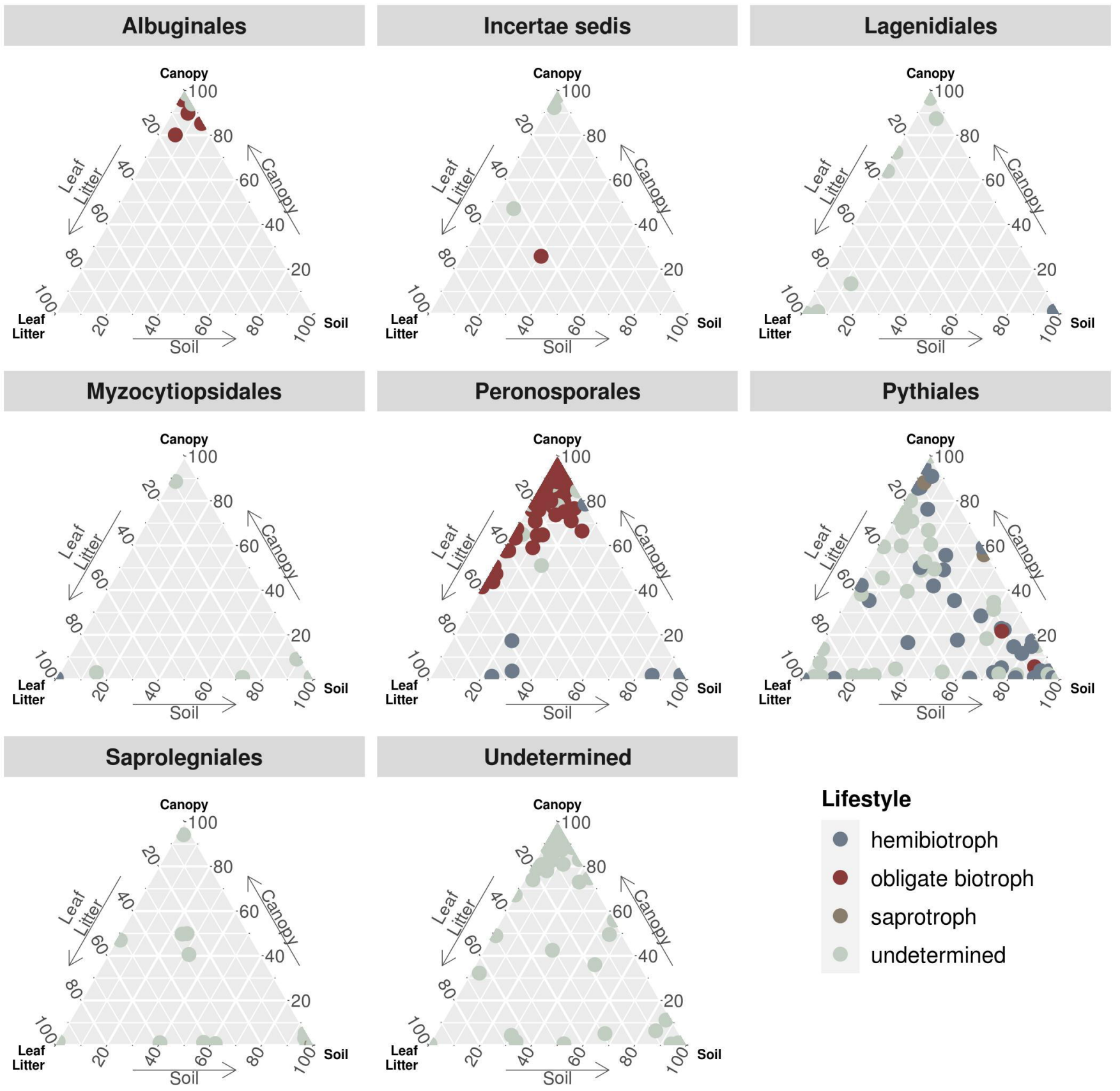




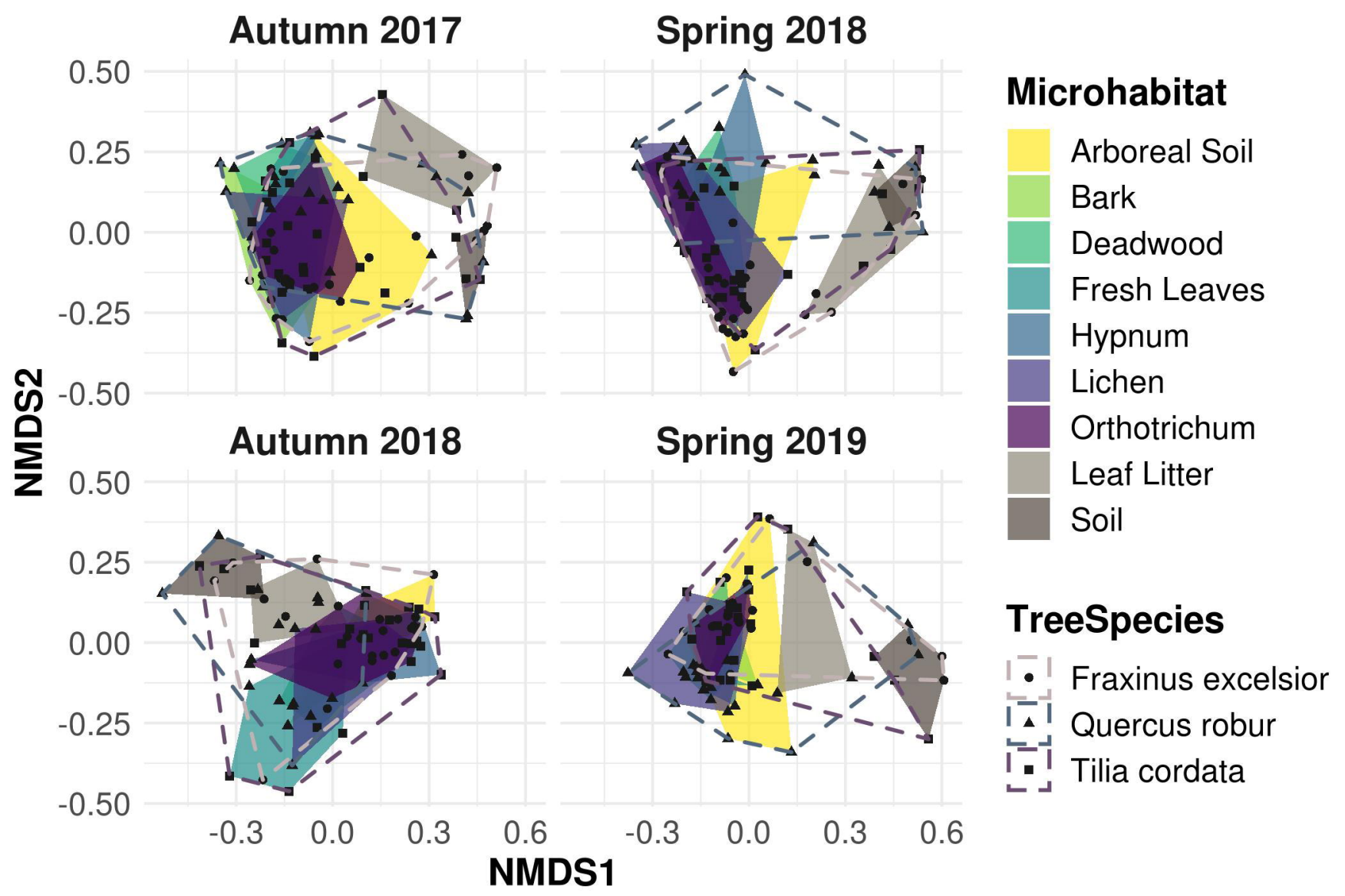

